\title{
Prevalence Of Risk Factors Associated With Ocular Problems In Males And Females In Type 1 \& 2 Diabetes
}

\author{
Ruqaya $^{1}$, Tayaba Kazmi ${ }^{2}$, Tehreem Anis ${ }^{3}$, Iqra Salahuddin ${ }^{4,}$ Moomal Zeshan ${ }^{4}$, Wajiha \\ Israr $^{1}$ \\ ${ }^{1}$ Department of Physiology, Baqai Medical University, Karachi, Pakistan. \\ ${ }^{2}$ Department of Anatomy, Baqai Medical University, Karachi, Pakistan. \\ ${ }^{3}$ Department of Physiotherapy, Pakistan Institute of Rehabilitation and Medical Sciences, Karachi, \\ Pakistan. \\ ${ }^{4}$ Department of Physical Rehabilitation, Pakistan Institute of Rehabilitation and Medical Sciences, \\ Karachi, Pakistan.
}

Received: June 6, 2021 Accepted: June 28, 2021

\begin{abstract}
Background: The aim of current study was exploring the ocular abnormalities in individuals with Type $1 \& 2$ diabetes. Methods: Patients referred for diabetic ocular abnormalities (retinopathy, cataract, glaucoma and refractive error) on fundoscopy from LRBT eye hospital Korangi, Karachi were randomly included in the study. Retinopathy, cataract and refractive error were evaluated by slit lamp biomicroscope or indirect ophthalmoscope, \& categorized into lacking, nonproliferative, proliferative retinopathy, maculopathy, capsular or sub capsular cataract, myopia and hypermetropia. While glaucoma was assessed by tonometry. Prevalence of eye complication has been seen in subjects. Outcome of substantial risk factors on the diverse phases of retinopathy, multivariate regression analysis was performed. Results: 200 diabetic subjects (106 males and 94 females) when the compared; diabetic subjects and subjects with hypertension; $28.0 \%$ retinopathy, 9.75 $\%$ cataract, $7.31 \%$ with glaucoma, and about $2.43 \%$ refractive error were found out among the diabetic males. $47.61 \%$ retinopathy, $11.9 \%$ Cataract, $40.47 \%$ refractive error were found in females. In males with diabetes and hypertension were found $60.46 \%$ retinopathy, $27.95 \%$ cataract, $11.62 \%$ refractive error \& in females $46.15 \%$ retinopathy, $19.23 \%$ cataract, and $34.61 \%$ with refractive error. Conclusions: This current study emphasizes screening of diabetic subjects regularly so as to distinguish ocular abnormalities and increasing public awareness.
\end{abstract}

Keywords: Type 1 diabetes mellitus, Type 2 Diabetes Mellitus, Retinopathy.

*Correspondence: Dr. Ruqaya Bux, Associate Professor, Department of Physiology, Baqai Medical University, Karachi Pakistan, Tel: +92-333-3362707 Email: ruqnanrs2009@yahoo.com

\section{Introduction}

Pakistan is a country in South Asia with an area of 796,095 square kilometers and a population of 207.7 million. [1] In terms of population, Pakistan is the sixth most populous country and the $36^{\text {th }}$ largest by geography in the world. Before 2018, the sole previous national diabetes study in Pakistan in 1999 (released in 2007), using the oral glucose tolerance test, the prevalence of type 2 diabetes was estimated to be $11 \%$ (OGTT).[2, 3] A part of an equivalent the prevalence of type 2 diabetes was reported independently in many Pakistani provinces in a survey. [2-5] The International Diabetes Federation (IDF) estimated the prevalence of diabetes in Pakistan to be $6.8 \%$ among people aged 20 to 79 years in its Atlas $5^{\text {th }}$ edition, [7] but health care practitioners with local knowledge always believed this to be an underestimate. As a result, there have been some discrepancies in prevalence findings varying from 7.2 percent to 19.21 percent in several parts of the country. [5]

Diabetic patients often develop ocular illnesses as a complication. However the foremost common and favorite risk factor among these complications is Diabetic Retinopathy (DR). [6-8] Albeit treatment of diabetic retinopathy can reduce the danger of visual loss by $60 \%$, diabetic retinopathy still remains the leading explanation for blindness among working-age adults. 
Diabetic retinopathy has been the primary focus due to its severe repercussions, which can result in premature blindness. This review deviates from a 'retinocentric' perspective by highlighting disorders other than diabetic retinopathy and macular edema that cause vision loss. Several studies have previously shown a link between diabetes and cataracts, glaucoma and age related macular degeneration (AMD), few people have thought about the visual issues that come with changes in refractive error, contrast sensitivity, stray light and presbyopia. These 'non-retinal' vision abnormalities could also be a symptom of severe hyperglycemia, especially when diabetes is diagnosed. Indeed, people with Type 2 diabetes are 1.4 times more likely than older persons without Type 2 diabetes to have these abnormalities, which can have a major impact on a person's quality of life. [8]

The start and progression of Diabetic Retinopathy (DR) are slow and progressive, progression from mild non-proliferative DR (NPDR), which is defined by the presence of at least one micro aneurysm, to moderate NPDR, which is defined by hemorrhages, micro aneurysms, and hard exudates. Hemorrhages and micro aneurysms in four quadrants, venous beading in at least two quadrants, and intraretinal microvascular anomalies in at least one quadrant, describe the severe type, neovascularization, preretinal hemorrhages, hemorrhage into the vitreous, traction retinal detachments, or macular edema (ME) are all symptoms of Proliferative Diabetes Retinopathy (PDR). [9]

There is a significant disparity in the reported prevalence of Diabetic Retinopathy. The global prevalence of DR among people with type 2 diabetes is 27.23 percent, although the prevalence of diabetes retinopathy varies greatly among ethnic groups, ranging from 20.8 percent among Asians to 46.7 percent among Caucasians. [10] The current study was carried to obtain the prevalence of eye complication among the diabetic individuals of Korangi.

Hypothesis: Risk factors have strong association with ocular problems in males and females in type $1 \& 2$ diabetes

\section{Materials \& Methods}

Study Design: The cross sectional study was carried out on diabetic patients referred for suspected diabetic eye complications like retinopathy, cataract, glaucoma and refractive error on fundoscopy from LRBT eye hospital Korangi, Karachi. Informed Consent and questionnaire was obtained from each participants including the information regarding familial history related to the pathogenesis of type 2 diabetes mellitus were obtained confidentially which included personal details, medical record, disease history, socio-economic status, diet and physical activities.

Inclusion criteria: The individuals with type $1 \& 2$ diabetes mellitus with the age ranging from 25-60 years were included in the study.

Exclusion criteria: Individuals with gestational diabetes and ages less than 25 were excluded from diabetes.

There are so many methods to diagnose diabetic eye complications. For this purpose various ophthalmic equipment are being used.

Optometry: During optometric/ophthalmological operations, the slit lamp bio-microscope was used. Ocular anoxia, posterior segment and optic area was visualized together with refraction of error. Binocular slit-lamp examination and a lens gave an expanded vision in detail, supporting anatomical diagnosis for a different eye disorders. [11] Optometrist examines the eye with the biomicroscope. To facilitate examination, stained a fine paper with a fluorescent dye which touched to eye on one side; this colors the tear film on the surface of the eye. Tears naturally wash color out of the eye. Following that, drops may be placed in the eyes the pupils dilation. 15 to 20 minutes of time taken by drops to yield effect, following the inspection was frequent to examine the eye. Patients will sensitive to light after few hours of examination, \& dilating drops increases pressure in eye, resulting in pain \& nausea. Subjects develop severe signs should seek medical 
assistance right away.[12] Adults do not require any extra grounding for test; but, children may require few changes according to their previous experiences, age $\&$ degree of trust.

\section{Ophthalmoscope}

Ophthalmoscopy (fundoscopy) is a test that uses an ophthalmoscope to allow a medical practitioner performs fundoscopy to view surrounding structures. It is performed as part of a standard physical checkup or determining the retinal and vitreous humour health. [13] It is classified into two categories. Indirect ophthalmoscopy generates reversed direct picture magnification two to five times and direct ophthalmoscopy generates an erect, or unreversed, picture of around magnification fifteen times.

It perceives \& assess retinal detachment or illnesses symptoms like swollen optic discs, glaucoma or papilledema, on ophthalmoscopy are critical indicator in patients with headaches, as this implies elevated intracranial pressure, which can cause hydrocephalus, benign intracranial hypertension, or a brain tumor, among other disorders. Glaucoma is characterized by cupped optic discs. Consistent eye examinations are crucial in individuals with diabetes mellitus to monitor for diabetic retinopathy, Hypertensive alterations in the retina closely match those in the brain in arterial hypertension and may precede cerebrovascular accidents (strokes). Ophthalmoscopy was thought to be 90-95 percent accurate in detecting the early stages and effects of many major disorders.[14]

Statistical Analysis: The baseline and clinical parameters was analyzed statistically using independent sample student-t test. Demographic parameters were depicted for graphical representation using MS Office \& SPSS version 16.0

\section{Results}

Table-1 showing prevalence of risk factors associated with ocular problems in males \& females. Male have comparatively more ocular problems associated with type $1 \& 2$ diabetic subjects.

\begin{tabular}{|c|c|c|c|c|c|c|}
\hline \multicolumn{7}{|c|}{ Table-1: Prevalence Of Risk Factors Associated With Ocular Problems In Males \& Females } \\
\hline \multicolumn{2}{|c|}{ Type 1 Diabetes N=53 } & \multicolumn{1}{l|}{ Type 2 Diabetes N=147 } \\
\hline \multirow{2}{*}{ Factors } & Male n=33 & Female n=20 & Total & $\begin{array}{c}\text { Male } \\
\text { n=73 }\end{array}$ & $\begin{array}{c}\text { Female } \\
\text { n=74 }\end{array}$ & Total \\
\hline \multirow{2}{*}{ HTN } & 17 & 13 & 30 & 29 & 36 & 65 \\
& $(51.5 \%)$ & $(65 \%)$ & $(62.26)$ & $(39.7 \%)$ & $(48.6 \%)$ & $(45.2 \%)$ \\
\hline \multirow{2}{*}{ Heart Attack } & 4 & 6 & 10 & 13 & 13 & 26 \\
& $(12.12 \%)$ & $(30 \%)$ & $(18.86 \%)$ & $(17.8 \%)$ & $(17.56 \%)$ & $(17.6 \%)$ \\
\hline $\begin{array}{c}\text { Hyper } \\
\text { cholesterolaemia }\end{array}$ & 5 & 5 & 10 & 13 & 24 & 37 \\
\hline \multirow{2}{*}{ Kidney Disease } & $(15.15 \%)$ & $(25 \%)$ & $(18.86 \%)$ & $(17.8 \%)$ & $(32.8 \%)$ & $(25.17 \%)$ \\
\hline \multirow{2}{*}{ F/H Diabetes } & $(39.39 \%)$ & $(50 \%)$ & $(43.39 \%)$ & $(32.8 \%)$ & $(25.6 \%)$ & $(29.2 \%)$ \\
\hline
\end{tabular}




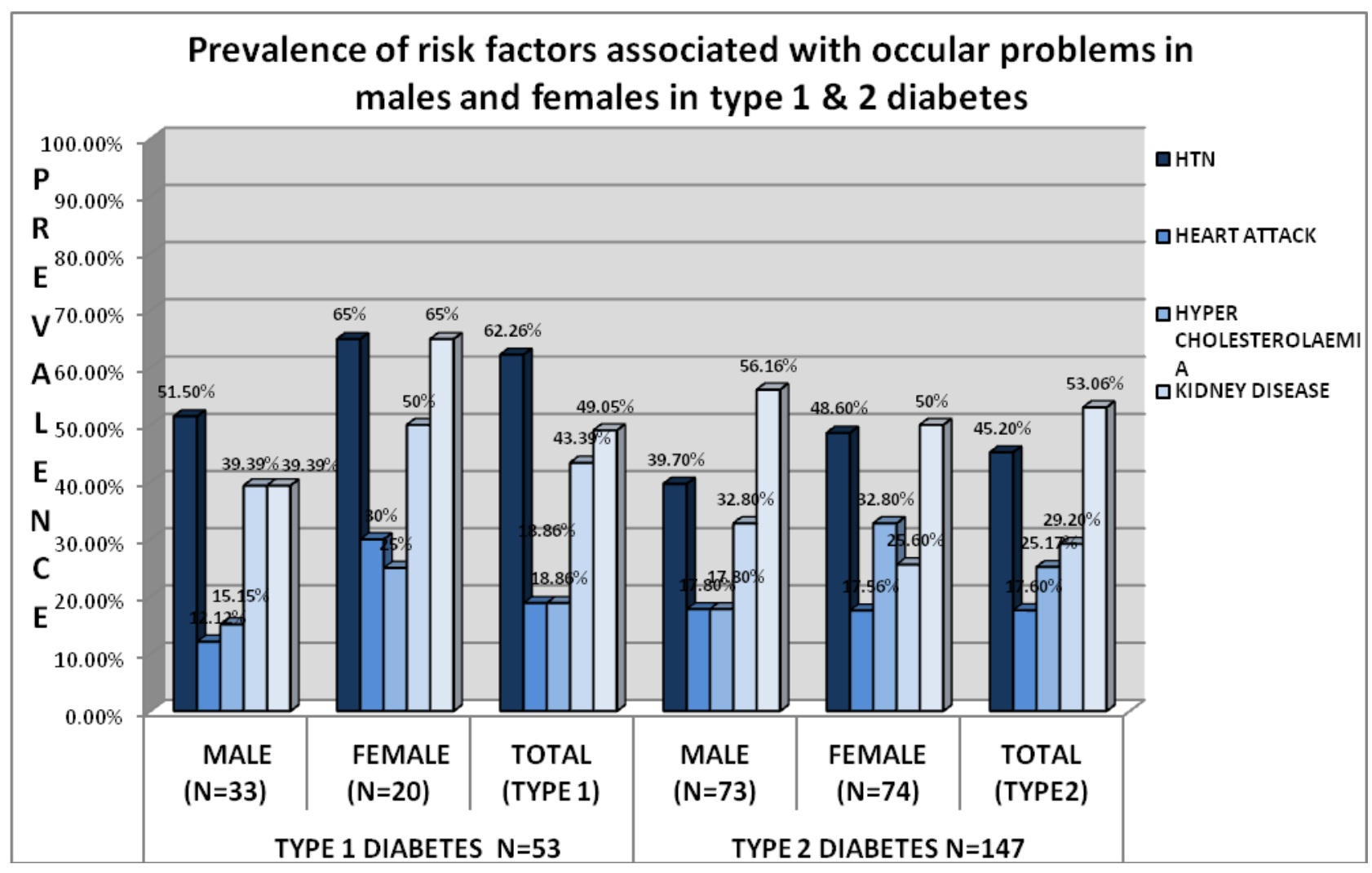

Figure 1 showing graphical representation of prevalence of risk factors associated with ocular problems in males and females in type $1 \& 2$ diabetes

\section{Discussion}

Diabetes mellitus is one of the growing epidemic globally, while Pakistan too experiences quite an alarming increase in the prevalence of the disease. The figure released by the International Diabetes federation in 2019 it was seen that about 19 million adults $(17.1 \%)$ in Pakistan are living with diabetes, putting them at the extreme risk of life threatening complications.[15] Our study was thus focused on estimating the prevalence and risk factors associated for eye complications in diabetic population.

Suspected diabetic eye complications such as retinopathy, cataract, glaucoma and refractive error on fundoscopy were evaluated on 200 subjects with 106 diabetic male ( $n=33$ type I, $n=73$ type II) and 94 ( $\mathrm{n}=20$ type I, n=74 type II) diabetic female subjects respectively. Our results showed a high prevalence male having retinopathy associated with both hypertension and diabetes type I (28.0\%) and type II (60.46\%) while in diabetic type I females had the occurrence of $47.61 \%$ cases and type II showed $46.15 \%$ cases of retinopathy which is similar to the study conducted on 1008 diabetic patients showing that about $40 \%$ of the population had some form of diabetic radiculopathy among which mostly being elderly hypertensive male. While our study also evaluated other ocular conditions that occur due to diabetes, in which among male subjects with type I diabetes $9.75 \%$ cases of cataract, $7.31 \%$ of glaucoma and $2.43 \%$ of refractive error cases were reported. While in the type I diabetic female subjects, Retinopathy was diagnosed in $47.61 \%$ of cases, while cataract was identified in $11.9 \%$ cases, and $40.47 \%$ were having refractive error. Subjects of type II diabetes were similarly tested and reported that among male subjects $27.95 \%$ had cataract, $11.62 \%$ were having refractive error. Whereas among the female subjects $19.23 \%$ were reported to have cataract and $34.61 \%$ were suffering with refractive error. 
Conclusion: Routinely visit is necessary to screen out the all the symptoms within due course of time; so that treatment may be initiated.

\section{ETHICS APPROVAL AND CONSENT TO PARTICIPATE}

Not applicable.

\section{HUMAN AND ANIMAL RIGHTS}

Not applicable.

\section{CONSENT FOR PUBLICATION}

Not applicable.

\section{AVAILABILITY OF DATA AND MATERIALS \\ None.}

\section{FUNDING}

None.

\section{CONFLICT OF INTEREST}

The authors declare no conflict of interest, financial or otherwise.

\section{ACKNOWLEDGEMENTS}

None.

\section{References}

1. Shera AS, Jawad F, Maqsood A. Prevalence of diabetes in Pakistan. Diabetes Res Clin Pract. 2007; 76(2):219-22.

2. Whiting DR, Guariguata L, Weil C, Shaw J. IDF diabetes atlas: global estimates of the prevalence of diabetes for 2011 and 2030. Diabetes Res Clin Pract. 2011; 94(3):311-21.

3. Schwartz AV, Vittinghoff E, Sellmeyer DE, Feingold KR, Rekeneire Nd, Strotmeyer ES et al. Diabetes-related complications, glycemic control, and falls in older adults. Diabetes Care 2008; 31: 391-396

4. El-Bab MF, Shawky N, Al-Sisi A, Akhtar M. Retinopathy and risk factors in diabetic patients from Al-Madinah Al-Munawarah in the Kingdom of Saudi Arabia. Clin Ophthalmol. 2012; 6:269-76.

5. Alberti KG, Zimmet PZ: Definition, diagnosis and classification of diabetes mellitus and its complications. Part 1: diagnosis and classification of diabetes mellitus provisional report of a WHO consultation. Diabet Med. 1998, 15:539-553. doi. 10.1002/(SICI)10969136(199807)15:7<539::AID-DIA668>3.0.CO;2-S

6. NCD Risk Factor Collaboration (NCD-RisC): Worldwide trends in diabetes since 1980: a pooled analysis of 751 population-based studies with 4.4 million participants. Lancet. 2016, 387:1513-1530. 10.1016/S0140-6736(16)00618-8.

7. Cai X, McGinnis JF. Diabetic retinopathy: Animal models, therapies, and perspectives. $J$ Diabetes Res. 2016:3789217.

8. Yau JW, Rogers SL, Kawasaki R, Lamoureux EL, Kowalski JW, Bek T, Chen SJ, Dekker et al.Global prevalence and major risk factors of diabetic retinopathy Meta-Analysis for Eye Disease (META-EYE) Study Group. Diabetes Care. 2012; 35(3):556-64.

9. Giloyan A, Harutyunyan T, Petrosyan V. The prevalence of and major risk factors associated with diabetic retinopathy in Gegharkunik province of Armenia: cross-sectional study. BMC Ophthalmol. 2015;15:46. doi: 10.1186/s12886-015-0032-0. 
10. Xu Z, Jiang H, Tao A, Wu S, Yan W, Yuan J, et al. Measurement variability of the bulbar conjunctival microvasculature in healthy subjects using functional slit lamp biomicroscopy (FSLB). Microvasc Res. 2015;101:15-9.

11. Jiang H, Zhong J, Debuc DC, Tao A, Xu Z, Lam BL, et al. Functional slit lamp biomicroscopy for imaging bulbar conjunctival microvasculature in contact lens wearers. Microvasc Res. 2014;92:62-71.

12. Shi Y, Hu L, Chen W, Qu D, Jiang H, Wang J. Evaluated conjunctival blood flow velocity in daily contact lens wearers. Eye Contact Lens. 2018;44(Suppl1):S238-43.

13. Chen W, Batawi HI, Alava JR, Galor A, Yuan J, Sarantopoulos CD, et al. Bulbar conjunctival microvascular responses in dry eye. Ocul Surf. 2017; 15(2):193-201.

14. IDF Diabetes Atlas 9th edition 2019.

15. Giloyan A, Harutyunyan T, Petrosyan V. The prevalence of and major risk factors associated with diabetic retinopathy in Gegharkunik province of Armenia: cross-sectional study. BMC Ophthalmol. 2015;15:46. doi: 10.1186/s12886-015-0032-0. 